\title{
Algorithmic Aspects of Secure Computation and Communication
}

\author{
Matt Franklin (U.C. Davis)
}

\begin{abstract}
We survey some recent progress in the design of efficient protocols for secure computation and communication, in a variety of cryptographic settings. The common thread is the usefulness of interesting algorithmic methods originally developed for non-cryptographic applications. We also present some intriguing open problems for which new algorithmic ideas may be needed.
\end{abstract}

